\title{
Les Propositions Subordonnées Dans Le Roman Madame Bovary Par Gustave Flaubert
}

Endah Ayu Fitri $\bowtie$ Sri Rejeki Urip ${ }^{\bowtie}$ Anastasia Pudjitriherwanti

Département de la Langue et la Littérature Étrangère, Faculté des Langues et des Arts, Universitas Negeri Semarang, Indonesia

\begin{tabular}{l}
\hline Info d'article \\
\hline Histoire de l'Atrticle: \\
Reçu Janvier 2020 \\
Accepté Mars 2020 \\
Publié Mai 2020 \\
\hline Keywords: \\
Sentence, Complex Sentence, \\
subordination \\
\hline
\end{tabular}

\begin{abstract}
A complex sentence of French language can be defined as a sentence that has two or more clauses associated with the word marker and between clauses. The complex sentence consists of three types, these are coordination, subordination, and juxtaposition. Every types have different characteristics with each other. This research only be focused in complex sentence or subordination. Based on explanation, the purposes of this research is to find and identified the form and function of Compound Sentence in Roman Madame Bovary by Gustave Flaubert. In this research explain the form and fonction of complex sentence in french in Roman Madame Bovary by Gustave Flaubert. According to Martin and Lecomte (1962) the complex sentences is divided in six form; la proposition subordonnée complétive, la proposition subordonnée circonstancielle, la proposition subordonnée interrogatif, la proposition subordonnée relative, la proposition subordonnée infinitive, and la proposition subordonnée participe that every form have been a different fonction. In this research used two approaches: theoretical approach (syntactic approach) and methodological approach (descriptive analytic qualitative). The data used in the research is the data that indicated as complex sentence in French roman Madame Bovary by Gustave Flaubert. The method of providing the data used in this research is a method of reading the basic techniques of sadap and advanced techniques to refer to the freely limate (SBLC) and note-taking technique, while the method of analysis used in this research is a method Distribution with the use of basic technique is for direct elements (B-U-L) and continued with the advanced technique of the technique of lecithin. The steps done in this study are: collecting data, identifying and specifying sentences that are indicated as complex sentences and then putting them into corpus of data, reading the whole sentence that is indicated as a complex sentence, translating the entire sentence indicated as a complex sentence, and analyzing the form and function of a complex sentence. From the results of the study, it can be concluded that the most dominant form of complex sentence found is Proposition Subordonnée Circonstancielle, and the function of the most dominant complex sentence found is the complement of noun in Proposition Subordonnée Relative. Advice given from this reseacher is expected to provide ideas for students in foreign language and literature, especially the French Literature study program to conduct research on complex sentences in French with an angle different prespectives and styles of analysis.
\end{abstract}




\section{Extrait}

La phrase complexe en français contient deux ou plusieurs verbes conjugués, elle est donc composée de deux ou plusieurs propositions est un mot français invariable qui sert à lier des mots, ou des propositions. Cette phrase complexe se compose de trois types, c'est-à dire: la coordination, la subordination, et la juxtaposition. Chaque type des phrases complexes a une caractéristique qui le distingue des autres. Basée sur l'explication, le but de cette recherche est de trouver et d'identifier la nature et l'emploi des Les Propositions Subordonnées dans Le Roman Madame Bovary par Gustave Flaubert. Cette recherche parle des propositions subordonnées dans le roman Madame Bovary par Gustave Flaubert, consistent en la nature et la fonction de ces propositions subordonnées. Selon Martin et Lecomte (1962), la nature des propositions subordonnées se compose de six natures, c'est-à-dire : la proposition subordonnée complétive, la proposition subordonnée circonstancielle, la proposition subordonnée interrogatif, la proposition subordonnée relative, la proposition subordonnée infinitive, et la proposition subordonnée participe dont chacun a sa propre fonction. Cette recherche utilise deux approches, ce sont l'approche de théorétique (l'approche de la syntaxe) et l'approche de méthodologique (descriptive analytique qualitative). Les sources de données utilisées dans cette recherche sont les phrases indiquées comme les propositions subordonnées dans le roman Madame Bovary par Gustave Flaubert. La méthode de recueillir des données dans cette recherche est la méthode de simak (la méthode d'épargne) avec la technique de basse sadap (la technique de taraudage de langue) et les techniques avancées : la technique SBLC (la chercheuse ne participe pas à la conversation réelle) et la technique catat (la chercheuse a obtenu les données de prise de notes dans la carte de données). La méthode utilisée dans cette recherche est la méthode distributionnelle avec 1'utilisation de la technique de basse Bagi Unsur Langsung (B-U-L), et puis continuée par les techniques avancées, c'est la technique d'ellipses. Les étapes de cette recherche sont: Collecter des données, identifier et classifier des phrases indiquées comme les propositions subordonnées et ensuite les arranger dans le corpus de données, lire tous les phrases indiquées comme les proposition subordonnées, traduire tous les phrases indiquées comme les propositions subordonnées, et analyser de la nature et la fonction des propositions subordonnées. Du résultat de la recherche, on peut conclure que la nature de la proposition subordonnée la plus trouvée est proposition subordonnée circonstancielle, et la fonction de la proposition subordonnée la plus trouvée est complément du nom dans la proposition subordonnée relative. Il est prévu que cette recherche donne une idée à l'étudiant du département de langue et de littérature étrangère, particulièrement le programme d'étude de littérature française pour faire des recherches sur la proposition subordonnée dans la langue française avec une perspective et un style d'analyse différents.

(C) 2020 Universitas Negeri Semarang

\begin{tabular}{lc}
\hline Addresse: & ISSN 2252-6730 \\
Gedung B4 FBS Universitas Negeri Semarang & \\
Kampus Sekaran, Gunungpati, Semarang, 50229 &
\end{tabular}




\section{INTRODUCTION}

La langue peut être interprétée littéralement comme un moyen de communication pour tout le monde. Selon Chaer (1995:19), la langue est un moyen d'expression de nos idées, de nos pensées, et de nos sentiments se compose de la forme et la signification de la langue qui permet la communication entre les individus. L'une de branches linguistiques qui parle de la langue et la syntaxe. Ramlan (1981:1) exprime que la syntaxe est une branche de la linguistique qui parle du discours, des propositions et des phrases. En puis, Grevisse (2008:15) explique aussi que la syntaxe étudie les relations entre les mots dans la phrase: l'ordre des mots, l'accord sont des phénomènes de syntaxe.

Selon Grevisse (2008:221), la phrase est une forme linguistique indépendante, qui n'est pas incluse dans une forme linguistique plus large. Martin et Lecomte (1962: 178) divisent la phrase en trois types, ce sont, les propositions indépendantes, les propositions dépendantes (les propositions principales et les propositions subordonnées), et les phrases complexes (la juxtaposition, la coordination, et la subordination).

Dans cette recherche, la chercheuse parle seulement de la subordination dans la phrase complexe ou des propositions subordonnées. Selon Martin et Lecomte (1962), la nature des propositions subordonnées se compose de six natures, c'est-à-dire : la proposition subordonnée complétive, la proposition subordonnée circonstancielle, la proposition subordonnée interrogatif, la proposition subordonnée relative, la proposition subordonnée infinitive, et la proposition subordonnée participe. Martin et Lecomte (1962) divisent aussi la fonction des propositions subordonnées en six fonctions, ce sont la proposition subordonnée complétive, la proposition subordonnée circonstancielle, la proposition subordonnée interrogative, la proposition subordonnée relative, la proposition subordonnée infinitive et la proposition subordonnée participe.

La recherche des propositions subordonnées est très intéressante parce qu'il se trouve encore rarement des recherches sur les phrases complexes en français, en particulier les propositions subordonnées. Afin que la chercheuse puisse plus facilement de trouver et analyser ces propositions subordonnées, la chercheuse utilise le roman Madame Bovary par Gustave Flaubert comme l'objet de recherche car le roman Madame Bovary par Gustave Flaubert est un des romans français populaires et du résultat d'observation préliminaire, il y a beaucoup de propositions subordonnées trouvées dans le roman. En plus, la chercheuse aime les œuvres littéraires classiques qui décrivent la réalité de la vie à cette époque. Donc, la chercheuse a décidé de faire les analyses sur la nature et la fonction des propositions subordonnées dans le roman Madame Bovary par Gustave Flaubert.

\section{MÉTHODE DE RECHERCHE}

Cette recherche utilise deux approches, ce sont l'approche de théorétique (l'approche de la syntaxe) et l'approche de méthodologique (descriptive analytique qualitative). Les sources de données utilisées dans cette recherche sont les phrases indiquées comme les propositions subordonnées dans le roman Madame Bovary par Gustave Flaubert.

La méthode de recueillir des données dans cette recherche est la méthode simak (la méthode de lire attentivement) avec la technique de basse sadap (la technique de recorder) et les techniques avancées : la technique SBLC (la chercheuse ne participe pas à la conversation réelle) et la technique catat (la chercheuse a obtenu les données de prise de notes dans la carte de données).

La méthode utilisée dans cette recherche est la méthode distributionnelle avec l'utilisation de la technique de basse Bagi Unsur Langsung (la chercheuse a divisé les éléments de données directement) et puis continuée par la technique avancée, c'est la technique Lesap (la technique d'ellipses). Les étapes de cette recherche sont: Collecter des données, identifier et classifier des phrases indiquées comme les propositions subordonnées et ensuite les arranger dans le corpus de données, lire tous les phrases indiquées comme les proposition subordonnées, traduire tous les phrases indiquées comme les propositions subordonnées, et analyser de la nature et la fonction des propositions subordonnées. 
La méthode de présentation utilisée dans cette recherche est la méthode informelle car l'explication de l'analyse des données sera plus détaillée.

\section{RÉSULTAT ET DISCUSSION}

Dans l'analyse de la recherche, la chercheuse parle de la nature et la fonction des propositions subordonnées dans le roman Madame Bovary par Gustave Flaubert.

\section{La Nature et La Fonction Des Propositions Subordonnées dans Le Roman Madame Bovary par Gustave Flaubert}

Grevisse (2008) explique que pour analyser une phrase, il doit contenir une nature divisée en trois types, ce sont: la proposition subordonnée, la proposition principale et la proposition indépendante. La proposition subordonnée est une conjonction subordonnée, un mot contenant un mot relatif (un mot relatif), ou un mot interrogatif pour l'interrogation indirecte. La proposition principale contrôle la proposition subordonnée. La proposition indépendante n'est ni la proposition principale ni la proposition subordonnée.

Basée sur la théorie de Martin et Lecomte (1962: 181) qui a été expliqué sur le cadre théorique en chapitre II, il y a six natures des propositions subordonnées en français, c'est-à-dire: la proposition subordonnée complétive, la proposition circonstancielle conjonctive, la proposition subordonnée interrogative, la proposition subordonnée relative, la proposition subordonnée infinitive, et la proposition subordonnée participiale. Les six formes des propositions subordonnées ont chacune une fonction proposée par la théorie de Grevisse dans son livre intitulé Cours d'Analyse Grammaticale et la théorie de Mauger en Analyse logique. Cette recherche utilise 64 données contenant la nature et la fonction des propositions subordonnées.

Ce qui suit sont les résultats d'analyse de certaines données.

(1) "Alors, madame Bovary, dit-il enfin, faites excuse, mais le devoir avant tout, vous savez ; il faut que j'expédie mes garnements."

La proposition principale $\quad$ : il faut

(Madame Bovary/234)

La proposition subordonnée : que j'expédie mes garnements

Nature : La proposition subordonnée complétive

Fonction : Sujet réel de l'expression de il faut

C'est le résultat du donné $\mathrm{n}^{0} 1$. Dans la dernière phrase, il y a proposition subordonnée marquée par le mot subordonnant (que) après le verbe impersonnel (il faut) qui décrire la fonction de sujet réel car il dans ce donnée n'est pas un objet mais le sujet réel Donc, cette proposition subordonnée est la proposition subordonnée complétive qui a la fonction de sujet réel de l'expression de il faut.

(2) "Après qu'il eut offert un siège, il s'assit pour déjeuner, tout en s'excusant beaucoup de l'impolitesse."

(Madame Bovary/616)

La proposition principale : il s'assit pour déjeuner, tout en s'excusant beaucoup de l'impolitesse

La proposition subordonnée : après qu'il eut offert un siège

Nature : La Proposition subordonnée circonstancielle

Fonction : Complément circonstancielle de temps du verbe s'assit

Dans le donné $\mathrm{n}^{0} 2$, il y a une circonstancielle de temps marqué par le mot subordonnant (après que). Donc cette proposition subordonnée est la proposition subordonnée circonstancielle qui a la fonction du complément circonstancielle de temps du verbe s'assit. 


\section{CONCLUSION}

Basée sur l'analyse de recherche des Propositions Subordonnées dans Le Roman Madame Bovary par Gustave Flaubert, on peut tirer quelques conclusions comme suit:

La nature des propositions subodonnées est divisée en six, c'est-à-dire: la proposition subordonnée complétive, la proposition subordonnée circonstancielle, la proposition subordonnée interrogative, la proposition subordonnée relative, la proposition subordonnée infinitive, et la proposition subordonnée participe. De 64 données analysées, il y a cinq phrases qui sont les propositions subordonnées complétives conjonctives, 31 phrases qui sont les propositions subordonnées circonstancielles conjonctives, trois phrases qui sont les propositions subordonnées interrogatives, 13 phrases qui sont les propositions subordonnées relative, une phrase qui est la proposition subordonnée infinitive, une phrase qui est la proposition subordonnée participe, et dix phrases qui sont le mélange de ces propositions subordonnées.

Dans cette recherche il y a des fonctions des propositions subordonnées. Premièrement, la proposition subordonnée complétive qui a la fonction de Sujet Réel (une phrase), Complément d'Objet (cinq phrases), rôles accessoires de la complétive (aucun) et locutions adverbiales (aucun). Deuxièmement, la proposition subordonnée circonstancielle qui a la fonction de complément circonstancielle de temps (dix phrases), complément circonstancielle de cause (trois phrases), complément circonstancielle de but (quatre phrases), complément circonstancielle de conséquence (trois phrases), complément circonstancielle d'opposition ou concession (trois phrases), complément circonstancielle de condition (deux phrases), et complément circonstancielle de comparaison (deux phrases). Ensuite, il y a la proposition subordonnée interrogative qui a la fonction de subordonnée sujet (aucun), subordonnée apposition (aucun), et complément d'objet (trois phrases). Puis, il y a la proposition subordonnée relative qui a la fonction de complément du nom (13 phrases). Cinquièmement, la proposition subordonnée infinitive qui a la fonction de complément d'objet du verbe principal (une phrase). Finalement, il y a la proposition subordonnée participe qui a la fonction de complément circonstancielle de temps (aucun), complément circonstancielle de cause (aucun), complément circonstancielle de condition (aucun), et complément circonstancielle de concession (une phrase).

Donc, on peut conclure que les propositions subordonnées circonstancielles conjonctives et les propositions subordonnées relative qui ont la fonction de complément du nom sont les propositions subordonnées le plus souvent trouvées dans cette recherche.

\section{BIBLIOGRAPHIE}

Abu-Ssydeh, Abdul-Fattah dan Najib Jarad. 2015. Complexe Setences in English Legislative Textes: Pattern and Translation Strategies. Volume XVI. Page 77-110. UAE: University of Sharjah.

Amalia, Riezha. 2013. Analisis Kalimat Majemuk dalam Komik Schneewittchen: Tanz Im Wald pada Majalah Anak Disney Prinzessin Edisi Nomor Dua Tahun 2005 Ditinjau dari Aspek Sintaksis. Mémoire. Universitas Indonesia. Jakarta.

Baryadi, Pratomo. 2007. "Rutan Klausa dalam Kalimat Majemuk Subordinatif Bahasa Indonesia: Kajian dari Prespektif Sintaksis dan Wacana. Humaniora. Volume XIX. Nomor 3. Page 224-231. Yogyakarta: Universitas Sanata Dharma.

Chaer, Abdul. 1995. Pengantar Semantik Bahasa Indonesia. Jakarta: PT. Rineka Cipta.

Crocker, Mary E. Coffman. 2005. Schaum's Outline of French Grammar: Edisi Keempat. Jakarta: Erlangga.

Delatour, dkk. 2004. Grammaire Française. Paris: Harchette.

Flaubert, Gustave. 1929. Madame Bovary. Paris: Librairie de France.

Grevisse, Maurice. 1968. Cours d'Analyse Grammatical. Paris: Gembloux Éditions Duculot.

Grevisse, Maurice. 2005. Le Petit Grevisse: Grammaire Française. Bruxelle: De Boeck \& Larcier. 
Grevisse, Maurice dan André Goosse. 2008. Le Bon Usage $14^{e}$ édition. Paris: Duculot.

Gordon, Petter C. dan Mattew W. Lowder. 2012. Complexe Setence Processing: A Review of Theoretical Prespectives on the Comprehension of Realative Clauses. USA: University of North Carolina.

Ibtissam, Kalfali. 2003. Defaillance Linguistique dans L'Emploi de la Phrase Complexe:Vers Une Approche Syntaxique. Mémoire. Université Mohammed Khider, Biskra, Republique Algerienne Democratique.

Kommeri, Noora. 2009. Analyse des Difficultés des Finnophones dans la Maîtrise des Phrases Complexes Françaises dans Les Tets Yki. Mémoire de Master. Université de Jyväskylä, Romane.

Košařová, Ivana. 2017. Les Phrases Complexes: Juxtaposition, Coordination, et Subordination. Thesis. Západočeská: Univerzita v Plzni.

Kridalaksana, Harimurti. 2008. Kamus Linguistik. Jakarta: Gramedia Pustaka Utama.

Léon, Pierre, dan Parth Batt. 2005. Structure du Française Moderne: Introduction à l'Analyse Linguistique. Toronto: Canadian Scholar's Press Inc.

Lessard, G. 1996. Introduction à la Linguistique Française. Kingston: Queen's University.

Mahsun. 2005. Metode Penelitian Bahasa: Tahapan Strategi, Metode, dan Tekniknya. Jakarta: PT.Raja Grafindo Persada.

Martin, J dan J. Lecomte. 1962. Grammaire Française. Paris: Masson et $\mathrm{C}^{\mathrm{I}-\mathrm{E}}$.

Mauger. G. 1955. Cours de Langue et de Civilisation Française. Paris: Librairie Hachette.

Noviana, Rina Dian. 2011. Konjungsi Temporal Bahasa Perancis dalam Majalah Marie Claire. Mémoire. Universitas Negeri Semarang, Semarang.

Prastuti, Dwi. 2012. Kalimat Majemuk Subordinatif dengan Anak Kalimat Atributif dalam Bahasa Rusia. Mémoire. Universitas Padjadjaran, Bandung.

Rahayu, Siti Perdi. 2017. Sintaksis Bahasa Perancis. Yogyakarta: TamanPena.com.

Ramlan. 1981. Sintaksis. Yogyakarta : CV. Karyono.

Subiyantoro. 2003. "Relasi Kausal dalam Bahasa Perancis dan Bahasa Indonesia". Humaniora. Volume XV. Nomor 2. Page 146-153. Yogyakarta: Universitas Gajah Mada.

Sudaryanto. 1993. Metode dan Aneka Teknik Analisis Bahasa. Yogyakarta: Duta Wacana University Perss.

Yulanda, Silvana. 2015. Kalimat Majemuk dalam Novel Rantau I Muara dan Implikasinya sebagai Bahan Ajar. Lampung: Universitas Negeri Lampung.

Yunitamara, Shela. 2016. Deskripsi Kalimat Majemuk dalam Gambar Tampilan Blackberry Messenger sebagai Bahan Ajar Pelajaran Bahasa Indonesia di SMP. Skripsi. Universitas Muhammadiyah Surakarta, Surakarta. 